\title{
Earthquake Processes: Physical Modelling, Numerical Simulation and Data Analysis
}

\author{
PART II \\ Mitsuhiro Matsu'ura, ${ }^{1}$ Peter Mora, ${ }^{2}$ \\ Andrea Donnellan, ${ }^{3}$ and Xiang-ChU Yin $^{4}$
}

\section{Introduction}

In the last decade of the 20th century, there has been great progress in the physics of earthquake generation; that is, the introduction of laboratory-based fault constitutive laws as a basic equation governing earthquake rupture, quantitative description of tectonic loading driven by plate motion, and a microscopic approach to study fault zone processes. The fault constitutive law plays the role of an interface between microscopic processes in fault zones and macroscopic processes of a fault system, and the plate motion connects diverse crustal activities with mantle dynamics. The APEC Cooperation for Earthquake Simulation (ACES) aims to develop realistic computer simulation models for the complete earthquake generation process on the basis of microscopic physics in fault zones and macroscopic dynamics in the crust-mantle system, and to assimilate seismological and geodetical observations into such models. Simulation of the complete earthquake generation process is an ambitious challenge. Recent advances in high performance computer technology and numerical simulation methodology are bringing this vision within reach.

The inaugural workshop of ACES was held on January 31 to February 5, 1999 in Brisbane and Noosa, Queensland, Australia. Following the fruitful results

\footnotetext{
${ }^{1}$ Department of Earth and Planetary Science, The University of Tokyo, Bunkyo-ku, Tokyo 113-0033, Japan. E-mail: matsuura@eps.s.u-tokyo.ac.jp

${ }^{2}$ QUAKES, Department of Earth Sciences, The University of Queensland, 4072 Brisbane, Qld, Australia. E-mail: mora@quakes.uq.edu.au

3 Jet Propulsion Laboratory NASA, 4800 Oak Grove Drive, Pasadena, CA 91109-8099, U.S.A. E-mail: donnellan@jpl.nasa.gov

${ }^{4}$ Laboratory of Nonlinear Mechanics, Institute of Mechanics, China Academy of Sciences, Beijing 100080, China. E-mail: yinxc@btamail.net.cn
} 
in the inaugural workshop [1, 2], the 2nd ACES workshop took place on October 15-20, 2000 in Tokyo and Hakone, Japan. In this workshop, more than 100 researchers in earthquake physics and computational science from 10 countries participated to discuss the integrated simulation-based approach for understanding earthquake processes. The major theme of the 2nd workshop was "microscopic and macroscopic simulation of fault zone processes and evolution, earthquake generation and cycles, and fault system dynamics." The theme was addressed in a series of six regular sessions for microscopic simulation, scaling physics, earthquake generation and cycles, dynamic rupture and wave propagation, data assimilation and understanding, and model applications to earthquake hazard quantification, and an additional special session for collaborative software systems and models [3]. We publish an outcome of the workshop as a set of two special issues (Part I and Part II) of Pure and Applied Geophysics for the six regular sessions. Articles for the additional special session, which cover primarily computer science and computational algorithm, are published separately in Concurrency and Computation: Practice and Experience. The articles in these special issues present a cross-section of cutting-edge research in the field of computational earthquake physics.

Part I collects articles covering two categories; A) micro-physics of rupture and fault constitutive laws and B) dynamic rupture, wave propagation and strong ground motion. Part II gathers articles encompassing two other categories; A) earthquake cycles, crustal deformation and plate dynamics and B) seismicity change and its physical interpretation.

Part II-A assembles articles on earthquake cycles, crustal deformation and plate dynamics. These range from the 3-D simulations of earthquake generation cycles driven by plate motion and interseismic crustal deformation associated with plate subduction to the development of new methods for analyzing geophysical and geodetical data and new simulation algorithms for large amplitude folding and mantle convection with viscoelastic/brittle lithosphere. These results provide us with important elements to construct an integrated realistic simulation model of crustal deformation and earthquake cycles.

Part II-B collects articles on seismicity change and its physical interpretation. These span a theoretical study of accelerated seismic release on heterogeneous faults, numerical simulation of long-range automaton models of earthquakes, and various approaches to earthquake prediction based on underlying physical or combined statistical and physical models for seismicity change. These studies provide us with new and important aspects to understand physical mechanisms underlying spatial and temporal change in seismicity.

Finally, we wish to thank all the participants of the 2nd ACES workshop and the contributors to these special issues, and to gratefully acknowledge financial support for the workshop by STA, RIST, NASA, NSF, ACDISR, ARC, JSPS, SSJ, TMKMF, FUJITSU, HITACHI, IBM, and NEC. 


\section{REFERENCES}

[1] 1-st ACES Workshop Proceedings (1999), ed. Mora, P. (ACES, Brisbane, Australia, ISBN 1-86499121-6), 554 pp.

[2] Microscopic and Macroscopic Simulation: Towards Predictive Modelling of the Earthquake Process (2000), eds. Mora, P., Matsu'ura, M., Madariaga, R., and Minster, J-B., Pure and Applied Geophysics, Volume 157, Number 11/12, 1817-2383.

[3] 2-nd ACES Workshop Proceedings (2001), eds. Matsu'ura, M., Nakajima, K., and Mora, P. (ACES, Brisbane, Australia, ISBN 1-864-99510-6), 605 pp. 